\title{
Sustained-Release Steroids for the Treatment of Diabetic Macular Edema
}

\author{
Alejandra Daruich ${ }^{1}$ - Alexandre Matet $^{1}$ • Francine Behar-Cohen ${ }^{1,2,3,4}$
}

Published online: 22 September 2015

(C) Springer Science+Business Media New York 2015

\begin{abstract}
Glucocorticoids have been used for decades in the treatment of ocular disorders via topical, periocular, and more recently intravitreal routes. However, their exact mechanisms of action on ocular tissues remain imperfectly understood. Fortunately, two recently approved intravitreal sustained-release drug delivery systems have opened new perspectives for these very potent drugs. To date, among other retinal conditions, their label includes diabetic macular edema, for which a long-lasting therapeutic effect has been demonstrated both morphologically and functionally in several randomized clinical trials. The rate of ocular complications of intravitreal sustained-release steroids, mainly cataract formation and intraocular pressure elevation, is higher than with anti-vascular endothelial growth factor agents. Yet, a better understanding of the mechanisms underlying these adverse effects and the search for the minimal efficient dose should help optimize their therapeutic window.
\end{abstract}

This article is part of the Topical Collection on Microvascular Complications-Retinopathy

Alejandra Daruich and Alexandre Matet contributed equally to this work.

Francine Behar-Cohen

francine.beharcohen@fa2.ch

Alejandra Daruich

adaruich.matet@gmail.com

Alexandre Matet

alexmatet@gmail.com

1 Department of Ophthalmology, University of Lausanne, Jules-Gonin Eye Hospital, Fondation Asile des Aveugles, Avenue de France 15, 1004 Lausanne, Switzerland
Keywords Diabetes mellitus · Macular edema · Therapy · Glucocorticoids $\cdot$ Delayed-action preparations $\cdot$ Fluocinolone acetonide $\cdot$ Dexamethasone

\section{Introduction}

Systemic glucocorticoids must be administered with caution to diabetic patients because they alter the glycemic homeostasis $[1,2]$ inducing peripheral insulin resistance [3] together with a progressive failure of pancreatic $\beta$-cells [4]. Through binding to the vascular and kidney mineralocorticoid receptor $[5,6]$, they favor hypertension $[7,8]$, further increasing the metabolic and cardiovascular risk factors of the diabetic patients [9]. Yet, intraocular corticosteroids are paradoxically gaining momentum in the local ocular treatment of diabetic macular edema [10]. The eye being a confined environment, isolated from the systemic circulation by blood-ocular bar-
2 Sorbonne Universités, UPMC Université Paris 06, UMR 1138, Centre de Recherche des Cordeliers, 3 rue de l'Ecole de Médecine, 75006 Paris, France

3 INSERM, UMR 1138, Centre de Recherche des Cordeliers, 3 rue de l'Ecole de Médecine, 75006 Paris, France

4 Université Paris Descartes, Sorbonne Paris Cité, UMR 1138, Centre de Recherche des Cordeliers, 3 rue de l'Ecole de Médecine, 75006 Paris, France 
riers, no significant systemic diffusion of corticosteroids is measured following their local administration in the vitreous, preventing from the systemic complications of glucocorticoids. Classically, the corticosteroid family is classified by the potency, the mineralocorticoid-binding affinity, and the half-life of each molecule. Yet, these classifications are translated from systemic to ocular use without evidence that the drugs maintain their pharmacologic properties in the eye, which questions the relevance of such translations. For instance, the mineralocorticoid pathway activation in the eye is not taken into account and the rate of specific ocular side effects, such as ocular hypertension, glaucoma, and cataract, are not included in the classification. The anti-edematous mechanisms triggered by glucocorticoids in the macula are complex and multi-factorial. They exert an intense, widespectrum anti-inflammatory action and are potent vasoconstrictors. Additionally, they regulate the expression of junction proteins in endothelial cells $[11,12]$ and the expression and distribution of ion channels and water channels in retinal glial Müller cells (Fig. 1a) [13]. Interestingly, dexamethasone and triamcinolone exert a specific and differential regulation of $\mathrm{K}+$ inwardly rectifying channel 4.1 (Kir4.1) and Aquaporin 4 (AQP4) in retinal glial Müller cells, suggesting that the dose and type of corticosteroid may influence their anti-edematous properties [13].

Triamcinolone acetonide was the first glucocorticoid injected into the vitreous $[14,15]$. The preparations used initially were not developed nor approved for intraocular use, although there are now triamcinolone acetonide formulations that are approved for intraocular use. Due to its very high hydrophobicity, triamcinolone acetonide forms a solid crystalline aggregate in the vitreous, which allows a long-lasting effect. But, triamcinolone acetonide was never incorporated in a drug delivery system that could provide a controlled release of the drug. Moreover, a potential toxicity of triamcinolone acetonide and of the more hydrophobic dexamethasone has been observed experimentally in vitro and in vivo on retinal and vascular cells. After intravitreal administration in healthy rats and in a murine model of choroidal neovascularization, triamcinolone acetonide triggered vascular endothelial cell death. It also exerted a deleterious effect on retinal pigment epithelium and Müller cells via a caspase-independent, paraptotic process [16]. The direct application of triamcinolone acetonide on rat retinal explants confirmed its toxic effect on vascular endothelial cells through caspaseindependent mechanisms [17]. Indeed, the controversy on the toxicity of glucocorticoids on the retina is mostly due to the fact that corticoids exert toxicity through nonclassical pathways, undetected by routine toxicology methods [16]. Nevertheless, the more hydrophobic, the more toxic because intracellular penetration is higher. Clinically, no prospective study was designed to assess the retinal safety of triamcinolone acetonide. But, authors have reported visual loss with
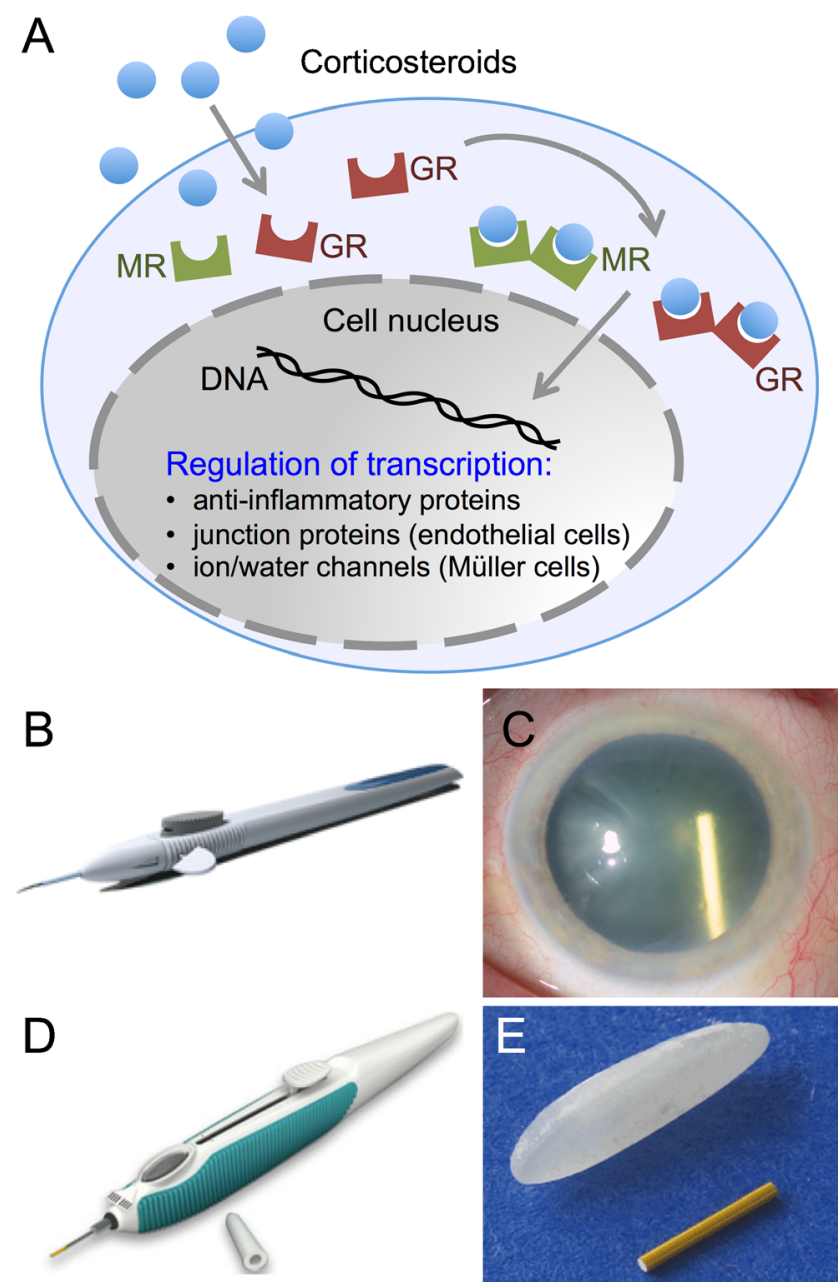

Fig. 1 a Schematic mechanism of corticosteroid action: after diffusion through the cell membrane, corticosteroids bind to the glucocorticoid (GR) and mineralocorticoid receptors (MR) according to their affinity profile and trigger a nuclear displacement of GR- and MR-homodimers that regulate the transcription of specific genes involved in the pathogenesis of macular edema. b Injection device for the dexamethasone implant $\left(\right.$ Ozurdex $\left.^{\mathbb{B}}\right)$. c Biomicroscopy photograph showing an intravitreal dexamethasone implant. d Injection device for the fluocinolone acetonide insert (Iluvien $^{\mathbb{B}}$ ). e Comparative photograph of the fluocinolone acetonide insert and a rice grain

electroretinographic alterations and visual field defects after repeated intravitreal triamcinolone injections [18], retinal pigment epithelium changes after accidental subretinal injection of triamcinolone [19], or optic atrophy after ILM peeling and intravitreal triamcinolone injection [20].

Recently, two drug delivery systems have been approved for the sustained intravitreal release of glucocorticoids, the dexamethasone (DEX) implant, and the fluocinolone acetonide (FAc) insert. Along with anti-vascular endothelial growth factor (VEGF) agents, they have expanded the toolbox available for retina specialists and have revolutionized the management of macular edema. However, none of them completely fulfills yet all optimal characteristics required by such therapeutic agents. This review will focus on the 
pharmacological specifications ideally required from such devices, the properties of the two approved products, and the clinical evidence regarding their therapeutic action and side effects.

\section{Pharmacological Requirements of an Ideal Sustained-Release System}

The advantages of drug delivery systems for the sustained release of corticosteroids are as follows:

- Controlled and sustained vitreous release of drug for an extended period of time - currently 3 months or more

- Reduction of the total administered dose with a potential reduction of ocular side effects

- Reduction of the frequency and cumulated number of intravitreal injections

- Improved pharmacokinetics with a flattening of concentration peaks and valleys

- Improvement of pharmacodynamic properties resulting from a controlled, stable concentration

An ideal sustained-release drug delivery system should present the following characteristics:

- Biodegradable matrix with a zero pharmacokinetic order.

- Sustained and controlled release lasting for an extended duration.

- Complete degradation of the matrix polymer when the entire drug is released.

- Biocompatibility and ocular tolerance of the matrix materials.

- Injectable device that fits in a small-gauge needle, typically 23 -gauge or thinner in the light of current standards for vitreoretinal procedures.

- Once injected, the implant should induce little or no visual disturbance.

- The implant design should allow potential removal in case of excessive side effects.

\section{Existing Steroid Sustained-Release Devices}

\section{Dexamethasone Intravitreal Implant}

The dexamethasone intravitreal implant (DEX implant, Ozurdex $^{\circledR}$; Allergan, Inc., Irvine, CA) consists of a sustained-release preparation containing $0.7 \mathrm{mg}$ of dexamethasone embedded in a biodegradable poly(lactic co-glycolic acid) matrix material (Fig. 1b, c). It has been designed to release dexamethasone into the vitreous for up to 6 months, with pharmacokinetics studies confirming the presence of the drug in the retina and vitreous of Macaca fascicularis monkeys 6 months after injection, with peak concentrations during the first 2 months, and undetectable levels after the sixth month [21]. However, clinical efficacy is observed up to 4 months after injection in some eyes [22•].

Dimensions of the DEX implant are as follows: length, $6 \mathrm{~mm}$, and diameter, $0.46 \mathrm{~mm}$, allowing it to fit into a specialized 22-gauge trans-scleral injector. Intravitreal injection is performed under topical anesthetic drops, and the selfsealing incision does not require sutures.

The DEX implant has been approved by the US Food and Drug Administration (FDA) for the following indications: diabetic macular edema (DME), macular edema following branch or central retinal vein occlusion, and noninfectious posterior uveitis.

\section{Sustained-Delivery Fluocinolone Acetonide Insert}

The sustained-delivery fluocinolone acetonide (FAc) insert (Iluvien ${ }^{\circledR}$; Alimera Sciences, Alpharetta, GA) is composed of nonbiodegradable cylindrical tubes of polymer loaded with $0.19 \mathrm{mg}$ of fluocinolone acetonide. The dimensions of the insert are $3.5 \times 0.37 \mathrm{~mm}$, and it is inserted into the vitreous via a 25 -gauge sutureless scleral incision using a manufactured injection device (Fig. 1d, e). It has been designed to release FAc at an initial rate of $0.25 \mu \mathrm{g} /$ day.

Aqueous humor levels of FAc peak during the first 3 months, followed by steady-state levels through 36 months [23]. These results are consistent with reports of clinical efficacy for up to 3 years post-insertion [24••].

The FDA approved the FAc insert for the treatment of DME in patients who have been previously treated with a course of corticosteroids and did not have a clinically significant rise in intraocular pressure.

\section{Main Outcomes of Clinical Trials}

For more than two decades from the mid-1980s, the standard of care for DME had been focal or grid laser photocoagulation associated to a strict control of glycemia, blood pressure, and other cardiovascular comorbidities [25]. Despite its widespread use from the mid-2000s and randomized clinical trials showing that it was beneficial for DME [26-28], the glucocorticoid triamcinolone acetonide was never formulated for intravitreal use specifically for DME treatment. Therefore, focal laser photocoagulation remained until recently the only approved therapeutic option for DME [29]. In 2012, ranibizumab, an anti-VEGF monoclonal antibody fragment, became the first approved drug for the treatment of DME, based on the results from the RIDE and RISE clinical trials $[30,31 \bullet \bullet]$, and proved superior to triamcinolone acetonide [32•]. This context explains why controlled, randomized clinical trials evaluating steroid-releasing devices have been designed differently according to the selected control 
intervention: sham, laser photocoagulation or anti-VEGF, with some trials also evaluating combinations of these treatments.

\section{Dexamethasone Intravitreal Implant}

The efficacy of the DEX implant has been assessed by several randomized clinical trials, which are summarized in Table 1.

In the randomized, masked, phase-III "MEAD" clinical trial that included 377 participants (cohort sizes and results mentioned for all clinical trials will refer to participants who completed the study until the end of the follow-up period), the 0.7-mg DEX implant was compared to sham over a 3-year period [33・0]. In a third arm, patients received DEX implants at a lower dose $(0.35 \mathrm{mg})$, but this formulation was eventually not retained for commercial distribution and will therefore not be mentioned further in this review. Noticeably, inclusion criteria required that baseline visual acuities should be between 20/200 and 20/50, thus excluding patients with good baseline visual acuity levels despite edema (a common finding in DME) and patients with very low baseline levels, who have usually less recovery potential. The study population was composed of a mixed cohort of treatment-naive patients and patients refractory to other therapies. Another specificity of the study design was the prohibition of focal laser treatment in the macula in both arms during the study period. The mean average reduction in central retinal thickness (measured at the fovea) from the baseline was greater in the DEX implant group, -112 versus $-42 \mu \mathrm{m}(p<0.001)$. The percentage of treated patients that had gained $\geq 15$ letters at 3 years was higher than in the sham group ( 22 vs $12 \%, p=0.018$ ). The mean visual acuity change over the 3 -year period was only + 3.5 letters for DEX-implanted patients, but this difference was significant compared to sham $(+2.0, p=0.023)$. Although not clinically meaningful for an individual eye, it indicates a favorable distribution of visual acuity gainers over losers among treated patients. In addition, $68 \%$ of subjects that were phakic at baseline had undergone cataract extraction at 3 years, versus $20 \%$ in the sham group. Noticeably, patients from the DEX group received a mean of only 4.1 injections over the 3 -year study period. Finally, the rates of dropout or loss to follow-up from this study were high (324 subjects out of 701 initially included in the $0.7 \mathrm{mg}$ DEX and sham arms) and could affect the interpretation of the results.

Another randomized, double-masked trial compared DEX implant and laser (103 participants) versus sham and laser (94 participants), with one possible retreatment by DEX/sham at 6 or 9 months, and three possible additional laser sessions [34]. In the DEX group, a significantly greater proportion of subjects had a visual improvement $\geq 10$ letters by 9 months, but this effect was not maintained at 12 months, suggesting that retreatment by DEX implant should be performed at intervals shorter than 6 months.

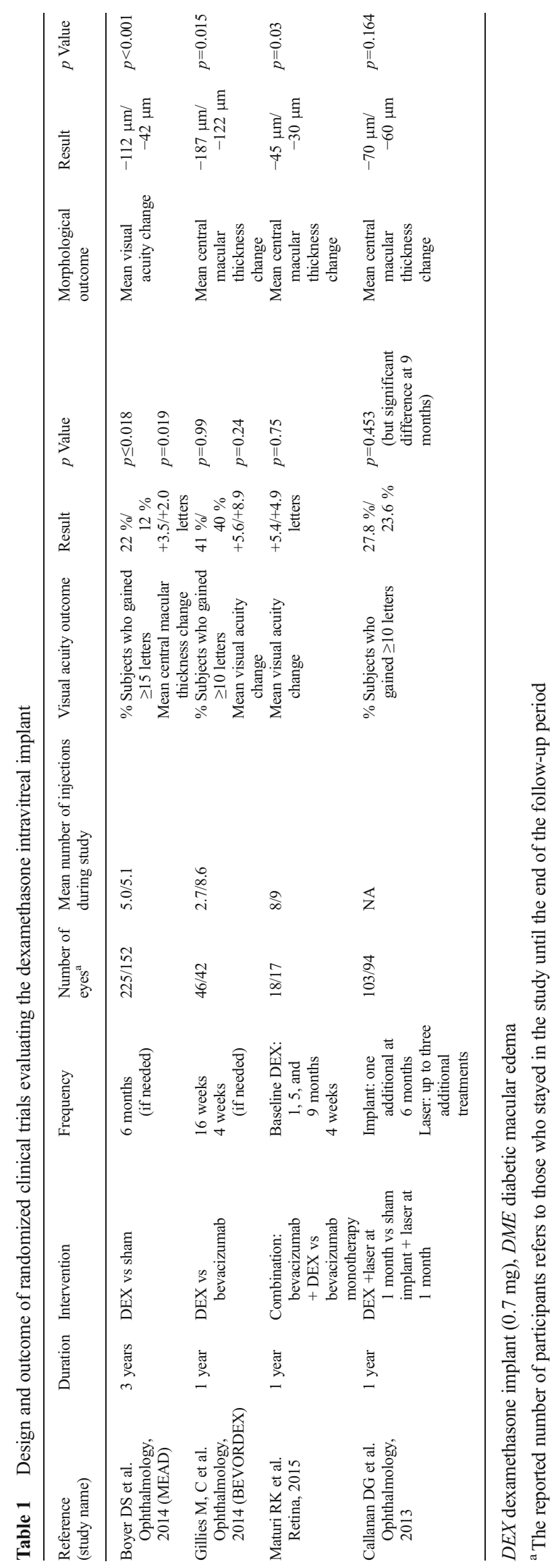


In the head-to-head single-masked, comparative, randomized "BEVORDEX" trial of DEX implant (46 subjects) versus the anti-VEGF antibody bevacizumab (42 subjects) [22•], both treatments achieved to improve visual acuity by 10 letters or more after 12 months in a similar proportion of subjects (40 and $41 \%$, respectively). A greater decrease in central macular thickness was observed in the DEX arm $(-187$ vs $-122 \mu \mathrm{m}$, $p=0.015$ ). Yet, five patients from the DEX group had a decrease in visual acuity, versus none from the bevacizumab group, a finding explained by the higher rate of cataract development in DEX-treated patients. Eventually, a mean of 2.7 DEX-implant injections versus 8.6 bevacizumab injections were performed over 12 months.

Next, a therapy combining bevacizumab at baseline and DEX implant at 1 month plus DEX implant re-injection at 5 and 9 months if needed (18 patients) has been evaluated versus bevacizumab monthly monotherapy (17 patients), in a randomized, single-masked design over 12 months [35]. There was a significant visual improvement in both groups from baseline but no difference in final visual acuities between groups. However, the mean central macular thickness reduction was greater in the combination group $(-45 \mathrm{vs}-30 \mu \mathrm{m} p=$ $0.03)$. In this group, the injection sparing effect was limited since patients received an average of eight additional injections compared to nine in the bevacizumab monotherapy group.

Interestingly, the efficacy of a single DEX implant over a 6month period has been reported in vitrectomized eyes [36, 37], although the absence of vitreous gel may have reduced the half-life of the drug within the vitreous cavity.

\section{Sustained-Delivery Fluocinolone Acetonide Insert}

Due to its more recent introduction and the longer study durations required, fewer studies have evaluated to date the FAc insert as compared to the DEX implant. Randomized clinical trials evaluating the FAc insert are summarized in Table 2. In the randomized, double-masked "FAME" trial [24・•] evaluating the FAc insert (releasing $0.25 \mu \mathrm{g} /$ day) (209 patients) against sham (112 patients), a gain in visual acuity $\geq 15$ letters was observed in $33 \%$ of FAc-treated subjects compared to $21 \%$ of sham-treated subjects after 3 years. Regarding the anatomical response, there was a significant decrease in central retinal thickness in the FAc-treated group compared to sham at 1 year, but no significant difference between the groups after 3 years.

A post hoc analysis of the previous study showed that the proportion of patients that gained $\geq 15$ letters of visual acuity was significantly greater in patients with DME of more than 3year duration treated by FAc versus sham, but such a difference was not observed in those with DME that lasted less than 3 years at the time of treatment [ $38 \bullet \bullet$. Other baseline characteristics did not differ between both groups.

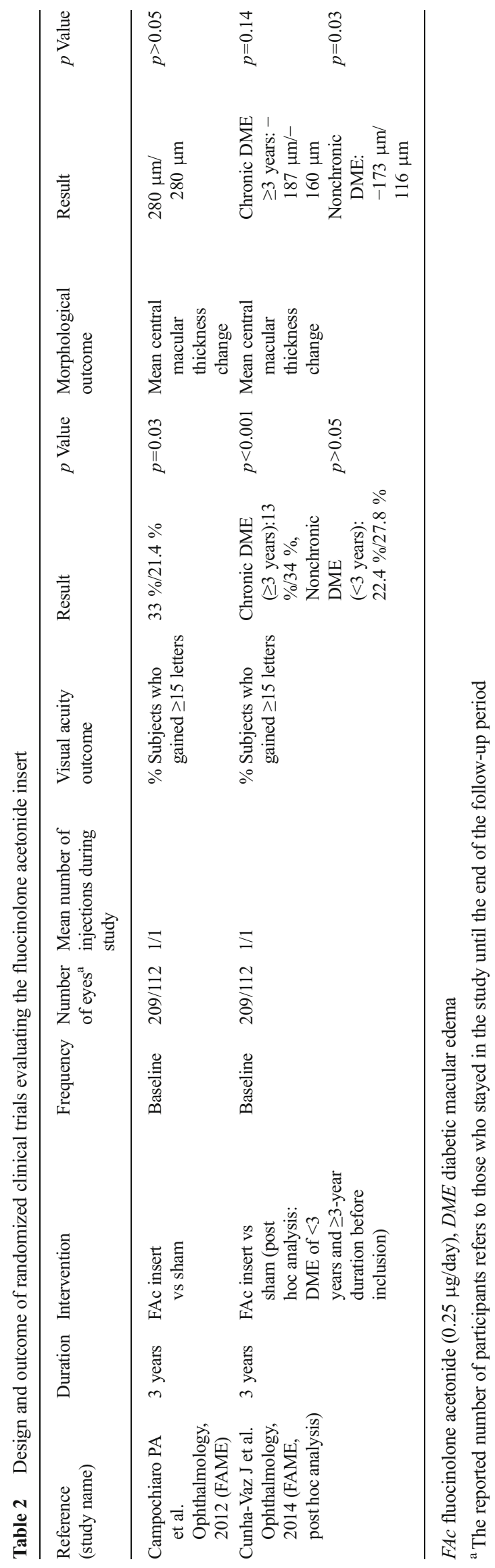




\section{Adverse Effects}

The most common ocular adverse effects of corticosteroids are secondary cataract formation and intraocular pressure rise. Both effects have been observed after topical and intraocular corticosteroids administration [39].

Despite its frequency, the mechanisms of steroid-induced lens opacification, most frequently posterior subcapsular, are not fully understood. It can develop after ocular but also systemic steroid treatment, and several possible pathways have been advanced [40]. Transcriptional changes may occur in lens epithelial cells, which express the nuclear glucocorticoid alpha receptor [41]. An imbalance in intraocular cytokines and growth factors affecting the lens homeostasis has also been suggested [42].

Corticosteroid-induced ocular hypertension results from an elevated resistance to aqueous outflow. Postulated mechanisms include: microstructural changes in the trabecular meshwork, deposition of precipitated substances in the trabecular meshwork, and inhibition of trabecular phagocytosis by endothelial cells contributing to this accumulation of substances [43].

Finally, a direct in vitro toxicity of corticosteroids on retinal vascular endothelial cells has been observed via autophagy, caspase-dependent and caspase-independent cell death, and direct DNA damage [17]. However, no acute retinal damages have been observed after intraocular steroids administration.

\section{Dexamethasone Intravitreal Implant}

Variable rates of cataract formation after DEX implant injection have been reported in the prospective studies described above, and range from 13 to $50 \%$ after 1 year [22•, 34, 35], and $68 \%$ after 3 years [33••]. In the trial by Boyer et al. with the longest study period, the rate of cataract extraction was $59 \%$ after 3 years in the DEX implant group versus $7 \%$ in the sham group.

In this trial, intraocular pressure rise over $25 \mathrm{mmHg}$ at any visit was observed in $32 \%$ of patients. Intraocular-pressurelowering medication was prescribed in $42 \%$ of subjects, but trabeculectomy was required in only $0.6 \%$ of cases. Other trials with follow-up of 1 year reported the occurrence of intraocular pressure rise over $25 \mathrm{mmHg}$ in $17-26 \%$ of cases $[22 \cdot, 34]$.

\section{Sustained-Delivery Fluocinolone Acetonide Insert}

In patients who received the FAc insert, cataract formation was observed in $82 \%$ over a 3-year period, with cataract extraction performed in $80 \%$ of FAc-implanted subjects versus $27 \%$ of sham-treated subjects [24••].

Among patients from this prospective cohort, $38 \%$ of subjects required intraocular-pressure-lowering medication
[24••]. Incisional glaucoma surgery was performed in $4.8 \%$ of FAc-treated subjects as compared to $0.5 \%$ of sham-treated subjects. Noticeably, the effect of FAc on intraocular pressure is likely to be dose-related, since a higher proportion of patients receiving the device releasing FAc $0.5 \mu \mathrm{g} / \mathrm{day}$ underwent cataract or glaucoma surgery ( 87 and $8 \%$, respectively).

\section{Conclusion}

Corticosteroids are among the most widely used classes of drugs in ophthalmology. With the advent of sustainedrelease devices, steroids are now also approved for the treatment of macular edema of various origins, including diabetic macular edema. Glucocorticoids and mineralocorticoids are expressed in retinal cells and in retinal pigment epithelial cells [44], and the expression of these receptors can be modulated in pathological states, as demonstrated in animal models [45]. When released in a sustained manner by intravitreal drug delivery systems, both dexamethasone and fluocinolone acetonides have a favorable effect on the course of diabetic macular edema. Yet, these long-lasting formulations have different pharmacological properties and side effect profiles. These differences can be, in part, explained by their different binding affinity to the glucocorticoid and mineralocorticoid receptors, subsequently leading to differential transcriptomic effects. Playing with these differential affinities, new steroids could be investigated that would optimize the clinical efficacy and reduce side effects. Among future optimization of these devices, the administered dose could be adapted to the disease state and a built-in, programmed dose tapering could reduce rebound effects.

These controlled-release ocular drug delivery systems have opened new applications for glucosteroids in ophthalmology, even though their mechanisms of action are not fully understood. They remain the object of intense investigations, in order to optimize this promising treatment strategy and expand it to other causes of macular edema.

\section{Compliance with Ethics Guidelines}

Conflict of Interest Alejandra Daruich, Alexandre Matet, and Francine Behar-Cohen declare that they have no conflict of interest.

Human and Animal Rights and Informed Consent This article does not contain any studies with human or animal subjects performed by any of the authors. 


\section{References}

Papers of particular interest, published recently, have been highlighted as:

- Of importance

•- Of major importance

1. Kwon S, Hermayer KL. Glucocorticoid-induced hyperglycemia. Am J Med Sci. 2013;345:274-7.

2. Perez A, Jansen-Chaparro S, Saigi I, Bernal-Lopez MR, Miñambres I, Gomez-Huelgas R. Glucocorticoid-induced hyperglycemia. J Diabetes. 2014;6:9-20.

3. Rafacho A, Ortsäter H, Nadal A, Quesada I. Glucocorticoid treatment and endocrine pancreas function: implications for glucose homeostasis, insulin resistance and diabetes. J Endocrinol. 2014;223:R49-62.

4. Rojas J, Chávez-Castillo M, Chávez-Castillo M, Cabrera M, Cabrera M, Bermúdez V, et al. Glucocorticoid-induced death of pancreatic Beta cells: an organized chaos. JOP. 2015;16:11-9.

5. Hawkins UA, Gomez-Sanchez EP, Gomez-Sanchez CM, GomezSanchez CE. The ubiquitous mineralocorticoid receptor: clinical implications. Curr Hypertens Rep. 2012;14:573-80.

6. Hunter RW, Bailey MA. Glucocorticoids and $11 \beta$-hydroxysteroid dehydrogenases: mechanisms for hypertension. Curr Opin Pharmacol. 2015;21:105-14.

7. Frey FJ, Odermatt A, Frey BM. Glucocorticoid-mediated mineralocorticoid receptor activation and hypertension. Curr Opin Nephrol Hypertens. 2004;13:451-8.

8. Goodwin JE, Geller DS. Glucocorticoid-induced hypertension. Pediatr Nephrol. 2012;27:1059-66.

9. Fardet L, Fève B. Systemic glucocorticoid therapy: a review of its metabolic and cardiovascular adverse events. Drugs. 2014;74: 1731-45.

10. Stewart MW. Corticosteroid use for diabetic macular edema: old fad or new trend? Curr Diab Rep. 2012;12:364-75.

11. Duh EJ. A novel mechanism for glucocorticoid-induced tightening of endothelial barriers. Invest Ophthalmol Vis Sci. 2013;54:4016.

12. Keil JM, Liu X, Antonetti DA. Glucocorticoid induction of occludin expression and endothelial barrier requires transcription factor p54 NONO. Invest Ophthalmol Vis Sci. 2013;54: 4007-15.

13. Zhao M, Bousquet E, Valamanesh F, Farman N, Jeanny J-C, Jaisser $\mathrm{F}$, et al. Differential regulations of AQP4 and Kir4.1 by triamcinolone acetonide and dexamethasone in the healthy and inflamed retina. Invest Ophthalmol Vis Sci. 2011;52:6340-7.

14. Jonas JB, Söfker A. Intraocular injection of crystalline cortisone as adjunctive treatment of diabetic macular edema. Am J Ophthalmol. $2001 ; 132: 425-7$

15. Martidis A, Duker JS, Greenberg PB, Rogers AH, Puliafito CA, Reichel E, et al. Intravitreal triamcinolone for refractory diabetic macular edema. Ophthalmology. 2002;109:920-7.

16. Torriglia A, Valamanesh F, Behar-Cohen F. On the retinal toxicity of intraocular glucocorticoids. Biochem Pharmacol. 2010;80:187886.

17. El Zaoui I, Behar-Cohen F, Torriglia A. Glucocorticoids exert direct toxicity on microvasculature: analysis of cell death mechanisms. Toxicol Sci. 2015;143:441-53.

18. Schulze-Döbold C, Weber M. Loss of visual function after repeated intravitreal injections of triamcinolone acetonide in refractory uveitic macular oedema. Int Ophthalmol. 2009;29:427-9.

19. Doshi A, Gariano RF. Visual loss associated with accidental subretinal injection of triamcinolone acetonide. Retin Cases Brief Rep. 2008;2:160-2.
20. Jaissle GB, Bartz-Schmidt KU, Szurman P. Optic atrophy subsequent to epiretinal triamcinolone deposits in an eye following inner limiting membrane peeling. Ophthalmologe. 2008;105:575-7.

21. Chang-Lin J-E, Attar M, Acheampong AA, Robinson MR, Whitcup SM, Kuppermann BD, et al. Pharmacokinetics and pharmacodynamics of a sustained-release dexamethasone intravitreal implant. Invest Ophthalmol Vis Sci. 2011;52:80-6.

22. Gillies MC, Lim LL, Campain A, Quin GJ, Salem W, Li J, et al. A randomized clinical trial of intravitreal bevacizumab versus intravitreal dexamethasone for diabetic macular edema: the BEVORDEX study. Ophthalmology. 2014;121:2473-81. This randomized trial demonstrates the injection-sparing benefit of long-lasting steroids compared to a.

23. Campochiaro PA, Nguyen QD, Hafiz G, Bloom S, Brown DM, Busquets M, et al. Aqueous levels of fluocinolone acetonide after administration of fluocinolone acetonide inserts or fluocinolone acetonide implants. Ophthalmology. 2013;120:583-7.

24.• Campochiaro PA, Brown DM, Pearson A, Chen S, Boyer D, RuizMoreno J, et al. Sustained delivery fluocinolone acetonide vitreous inserts provide benefit for at least 3 years in patients with diabetic macular edema. Ophthalmology. 2012;119:2125-32. This randomized trial presents encouraging long-term results for the fluocinolone acetonide insert.

25. Photocoagulation for diabetic macular edema. Early Treatment Diabetic Retinopathy Study report number 1. Early Treatment Diabetic Retinopathy Study research group. Arch. Ophthalmol. 1985;103:1796-806.

26. Diabetic Retinopathy Clinical Research Network. A randomized trial comparing intravitreal triamcinolone acetonide and focal/grid photocoagulation for diabetic macular edema. Ophthalmology. 2008;115:1447-9. 1449.e1-10.

27. Gillies MC, Sutter FKP, Simpson JM, Larsson J, Ali H, Zhu M. Intravitreal triamcinolone for refractory diabetic macular edema: two-year results of a double-masked, placebo-controlled, randomized clinical trial. Ophthalmology. 2006;113:1533-8.

28. Gillies MC, Simpson JM, Gaston C, Hunt G, Ali H, Zhu M, et al. Five-year results of a randomized trial with open-label extension of triamcinolone acetonide for refractory diabetic macular edema. Ophthalmology. 2009;116:2182-7.

29. Gunther J, Ip M. Intravitreal steroid versus macular laser for treatment of diabetic macular edema. Curr Diab Rep. 2009;9:272-6.

30. Nguyen QD, Brown DM, Marcus DM, Boyer DS, Patel S, Feiner L, et al. Ranibizumab for diabetic macular edema: results from 2 phase III randomized trials: RISE and RIDE. Ophthalmology. 2012;119: 789-801.

31.• Brown DM, Nguyen QD, Marcus DM, Boyer DS, Patel S, Feiner L, et al. Long-term outcomes of ranibizumab therapy for diabetic macular edema: the 36-month results from two phase III trials: RISE and RIDE. Ophthalmology. 2013;120:2013-22. Large-scale clinical trial demonstrating the superiority of the anti-VEGF ranibizumab over placebo in diabetic macular edema.

32. Elman MJ, Bressler NM, Qin H, Beck RW, Ferris FL, Friedman SM, et al. Expanded 2-year follow-up of ranibizumab plus prompt or deferred laser or triamcinolone plus prompt laser for diabetic macular edema. Ophthalmology. 2011;118:609-14.

33.•• Boyer DS, Yoon YH, Belfort R, Bandello F, Maturi RK, Augustin AJ, et al. Three-year, randomized, sham-controlled trial of dexamethasone intravitreal implant in patients with diabetic macular edema. Ophthalmology. 2014;121:1904-14. Large randomized trial demonstrating the superiority of sustained-release intravitreal dexamethasone over placebo for macular edema.

34. Callanan DG, Gupta S, Boyer DS, Ciulla TA, Singer MA, Kuppermann BD, et al. Dexamethasone intravitreal implant in combination with laser photocoagulation for the treatment of diffuse diabetic macular edema. Ophthalmology. 2013;120:1843-51. 
35. Maturi RK, Bleau L, Saunders J, Mubasher M, Stewart MW. (2015) A 12-month, single-masked, randomized controlled study of eyes with persistent diabetic macular edema after multiple anti-VEGF injections to assess the efficacy of the dexamethasone-delayed delivery system as an adjunct to bevacizumab compared with continued bevacizumab monotherapy. Retina.

36. Boyer DS, Faber D, Gupta S, Patel SS, Tabandeh H, Li X-Y, et al. Dexamethasone intravitreal implant for treatment of diabetic macular edema in vitrectomized patients. Retina. 2011;31:915-23.

37. Medeiros MD, Alkabes M, Navarro R, Garcia-Arumí J, Mateo C, Corcóstegui B. Dexamethasone intravitreal implant in vitrectomized versus nonvitrectomized eyes for treatment of patients with persistent diabetic macular edema. J Ocul Pharmacol Ther. 2014;30:709-16.

38.• Cunha-Vaz J, Ashton P, Iezzi R, Campochiaro P, Dugel PU, Holz FG, et al. Sustained delivery fluocinolone acetonide vitreous implants: long-term benefit in patients with chronic diabetic macular edema. Ophthalmology. 2014;121:1892-903. Post hoc study showing a greater benefit of fluocinolone acetonide insert in patient with chronic, refractory diabetic macular edema of 3 years duration or more.

39. Ciulla TA, Harris A, McIntyre N, Jonescu-Cuypers C. Treatment of diabetic macular edema with sustained-release glucocorticoids: intravitreal triamcinolone acetonide, dexamethasone implant, and fluocinolone acetonide implant. Expert Opin Pharmacother. 2014;15:953-9.

40. James ER. The etiology of steroid cataract. J Ocul Pharmacol Ther. 2007;23:403-20.

41. Stokes J, Noble J, Brett L, Phillips C, Seckl JR, O'Brien C, et al. Distribution of glucocorticoid and mineralocorticoid receptors and 11 beta-hydroxysteroid dehydrogenases in human and rat ocular tissues. Invest Ophthalmol Vis Sci. 2000;41:1629-38.

42. Jobling AI, Augusteyn RC. What causes steroid cataracts? A review of steroid-induced posterior subcapsular cataracts. Clin Exp Optom. 2002;85:61-75.

43. Jones R, Rhee DJ. Corticosteroid-induced ocular hypertension and glaucoma: a brief review and update of the literature. Curr Opin Ophthalmol. 2006;17:163-7.

44. Zhao M, Valamanesh F, Celerier I, Savoldelli M, Jonet L, Jeanny J$\mathrm{C}$, et al. The neuroretina is a novel mineralocorticoid target: aldosterone up-regulates ion and water channels in Müller glial cells. FASEB J. 2010;24:3405-15.

45. Bousquet E, Zhao M, Ly A, Leroux Les Jardins G, Goldenberg B, Naud M-C, et al. The aldosterone-mineralocorticoid receptor pathway exerts anti-inflammatory effects in endotoxin-induced uveitis. PLoS One. 2012;7, e49036. 